University of Nebraska - Lincoln

DigitalCommons@University of Nebraska - Lincoln

Faculty Publications from Nebraska Center for

Materials and Nanoscience, Nebraska Center Materials and Nanoscience

for (NCMN)

August 2007

\title{
Measurement of Poisson's ratio with contact-resonance atomic force microscopy
}

\author{
D.C. Hurley \\ Materials Reliability Division, National Institute of Standards \& Technology, Boulder, CO \\ Joseph A. Turner \\ University of Nebraska - Lincoln, jaturner@unl.edu
}

Follow this and additional works at: https://digitalcommons.unl.edu/cmrafacpub

Part of the Nanoscience and Nanotechnology Commons

Hurley, D.C. and Turner, Joseph A., "Measurement of Poisson's ratio with contact-resonance atomic force microscopy" (2007). Faculty Publications from Nebraska Center for Materials and Nanoscience. 40. https://digitalcommons.unl.edu/cmrafacpub/40

This Article is brought to you for free and open access by the Materials and Nanoscience, Nebraska Center for (NCMN) at DigitalCommons@University of Nebraska - Lincoln. It has been accepted for inclusion in Faculty Publications from Nebraska Center for Materials and Nanoscience by an authorized administrator of DigitalCommons@University of Nebraska - Lincoln. 


\title{
Measurement of Poisson's ratio with contact-resonance atomic force microscopy
}

\author{
D. C. Hurley ${ }^{\text {a) }}$ \\ Materials Reliability Division, National Institute of Standards \& Technology, \\ Boulder, Colorado 80305, USA \\ J. A. Turner
Department of Engineering Mechanics, University of Nebraska, Lincoln, Nebraska 68588, USA
}

(Received 19 March 2007; accepted 23 June 2007; published online 9 August 2007)

\begin{abstract}
We describe contact-resonance atomic force microscopy (AFM) methods to quantitatively measure Poisson's ratio $\nu$ or shear modulus $G$ at the same time as Young's modulus $E$. In contact-resonance $\mathrm{AFM}$, the frequencies of the cantilever's resonant vibrations are measured while the tip is in contact with the sample. Simultaneous measurement of flexural and torsional vibrational modes enables $E$ and $\nu$ to be determined separately. Analysis methods are presented to relate the contact-resonance frequencies to the tip-sample contact stiffness, which in turn determines the sample's nanoscale elastic properties. Experimental results are presented for a glass specimen with fused silica used as a reference material. The agreement between our contact-resonance AFM measurements and values obtained from other means demonstrates the validity of the basic method. (C) 2007 American Institute of Physics. [DOI: 10.1063/1.2767387]
\end{abstract}

\section{INTRODUCTION}

Successful development of micro- and nanoscale devices requires the ability to characterize material properties with commensurate spatial resolution. For example, knowledge of small-scale mechanical properties such as modulus, friction, and adhesion is crucial. ${ }^{1,2}$ One widely established method to determine micro- and nanomechanical properties is depthsensing indentation ("nanoindentation"). ${ }^{3}$ Methods based on atomic force microscopy (AFM) provide even greater spatial resolution. Many AFM-based techniques, as well as nanoindentation, involve the contact mechanics between a probe tip and the sample of interest. In such contact methods, measurements yield a quantity $M$, the so-called indentation or plane-strain modulus. For isotropic materials, $M$ is actually a combination of two separate elastic properties, Young's modulus $E$ and Poisson's ratio $\nu: M=E /\left(1-\nu^{2}\right)$.

However, it is sometimes desirable to know the individual elastic properties separately. For instance, properties such as $\nu$ or the shear modulus $G$ are valuable in understanding thin-film behavior. ${ }^{4}$ A nanoindentation-based method for this purpose has been demonstrated that uses a custom apparatus with three-axis motion. ${ }^{5}$ AFM approaches that have been proposed to determine shear properties include passive overtone microscopy ${ }^{6}$ and a combination of modulated lateral force and magnetic force modulation microscopies. ${ }^{7}$

Another AFM-based method that provides nanomechanical information is atomic force acoustic microscopy (AFAM) ${ }^{8}$ AFAM is a type of "contact-resonance spectroscopy AFM," or more simply, "contact-resonance AFM," method. Such methods involve the resonant vibrational modes of the cantilever beam when the tip is in contact with

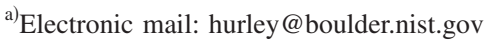

the sample. To date, methods such as AFAM have been used primarily to evaluate the indentation modulus $M$ by means of flexural cantilever modes. However, it has been noted that one might also utilize torsional cantilever modes to obtain additional elastic-property information. ${ }^{9,10}$ This idea was first noted several years ago, but to our knowledge it has not been experimentally demonstrated. Torsional or lateral AFM methods are typically used to study friction and dissipation. ${ }^{10-12}$ In this article, we describe experiments that involve the simultaneous measurement of flexural and torsional contact-resonance frequencies. We then show how these measurements can be used to obtain shear elastic properties such as $\nu$ or $G$ separately from $E$. These proof-ofprinciple experiments were not designed to demonstrate the nanoscale spatial resolution achievable with AFM methods. Nonetheless, they point the way toward quantitative nanomechanical measurements.

\section{THEORETICAL BACKGROUND}

In contact-resonance AFM, the resonant vibrational modes of the cantilever beam are excited by an external means. The frequency at which the resonance occurs is measured when the tip is in free space and when it is in contact with the sample of interest. As seen below, it is the difference between these "free-space" and "contact-resonance" frequencies that enables quantitative determination of the elastic properties of the sample. The two analytical models used to achieve this goal are summarized below. The first model describes the dynamic motion of the cantilever, and the second model involves the contact mechanics between the tip and the sample. 


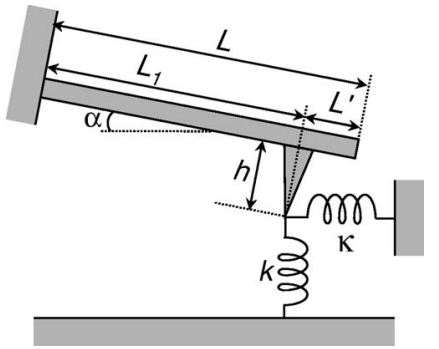

FIG. 1. Model for cantilever dynamics. The cantilever is a rectangular beam of length $L$ that is clamped at one end. The cantilever is tilted at an angle $\alpha$ with respect to the sample. The tip has length $h$ and is located at a position $L_{1}=L-L^{\prime}$ with respect to the clamped end. The tip is coupled to the sample by a vertical (normal) spring of stiffness $k$ and a horizontal (tangential) spring of stiffness $\kappa$.

\section{A. Cantilever dynamics}

Figure 1 illustrates the key components of the contactresonance model. The cantilever is modeled as a rectangular beam of uniform cross section with length $L$, width $w$, thickness $b$, and density $\rho$. The tip has height $h$ and is located at $L_{1}<L$. The remaining distance to the end of the cantilever is $L^{\prime}$, so that $L=L_{1}+L^{\prime}$. The flexural spring constant of the cantilever is $k_{\text {lever }}=E b^{3} w /\left(4 L_{1}^{3}\right)$, where $E$ is Young's modulus. The lateral stiffness is $\kappa_{\text {lever }}=w b^{3} G /\left(3 L h^{2}\right)$, where $G$ is the shear modulus. The cantilever is tilted by an angle $\alpha$ with respect to the sample surface. The elastic interaction between the tip and the sample is represented by two springs: a vertical (normal) spring with stiffness $k$, and a lateral (tangential) spring with stiffness $\kappa$.

The basic equations to describe this system have been previously published. ${ }^{13,14}$ Here, we state the final result in a form that lends itself to numerical solution. The vertical spring constant or contact stiffness $k$ normalized by the flexural cantilever stiffness $k_{\text {lever }}$ is given by the positive root of

$$
\frac{k}{k_{\text {lever }}}=\frac{-B \pm \sqrt{B^{2}-4 A C}}{6 A},
$$

where

$$
\begin{aligned}
A= & \left(\frac{\kappa}{k}\right)\left(\frac{h}{L_{1}}\right)^{2}\left(1-\cos x_{n} L_{1} \cosh x_{n} L_{1}\right) \\
& \times\left(1+\cos x_{n} L^{\prime} \cosh x_{n} L^{\prime}\right), \\
B= & B_{1}+B_{2}+B_{3}, \\
C= & 2\left(x_{n} L_{1}\right)^{4}\left(1+\cos x_{n} L \cosh x_{n} L\right),
\end{aligned}
$$

with

$$
\begin{aligned}
B_{1}= & \left(\frac{h}{L_{1}}\right)^{2}\left(x_{n} L_{1}\right)^{3}\left(\sin ^{2} \alpha+\frac{\kappa}{k} \cos ^{2} \alpha\right) \\
& \times\left[( 1 + \operatorname { c o s } x _ { n } L ^ { \prime } \operatorname { c o s h } x _ { n } L ^ { \prime } ) \left(\sin x_{n} L_{1} \cosh x_{n} L_{1}\right.\right. \\
& \left.+\cos x_{n} L_{1} \sinh x_{n} L_{1}\right)-\left(1-\cos x_{n} L_{1} \cosh x_{n} L_{1}\right) \\
& \left.\times\left(\sin x_{n} L^{\prime} \cosh x_{n} L^{\prime}+\cos x_{n} L^{\prime} \sinh x_{n} L^{\prime}\right)\right]
\end{aligned}
$$

$$
\begin{aligned}
B_{2}= & \left(\frac{h}{L_{1}}\right)\left(x_{n} L_{1}\right)^{2}\left[\left(\frac{\kappa}{k}-1\right) \cos \alpha \sin \alpha\right] \\
& \times\left[\left(1+\cos x_{n} L^{\prime} \cosh x_{n} L^{\prime}\right) \sin x_{n} L_{1} \sinh x_{n} L_{1}\right. \\
& \left.\left.+\left(1-\cos x_{n} L_{1} \cosh x_{n} L_{1}\right) \sin x_{n} L^{\prime} \sinh x_{n} L^{\prime}\right)\right], \\
B_{3}= & \left(x_{n} L_{1}\right)\left(\cos ^{2} \alpha+\frac{\kappa}{k} \sin ^{2} \alpha\right) \\
& \times\left[( 1 + \operatorname { c o s } x _ { n } L ^ { \prime } \operatorname { c o s h } x _ { n } L ^ { \prime } ) \left(\sin x_{n} L_{1} \cosh x_{n} L_{1}\right.\right. \\
& \left.-\cos x_{n} L_{1} \sinh x_{n} L_{1}\right)-\left(1-\cos x_{n} L_{1} \cosh x_{n} L_{1}\right) \\
& \left.\times\left(\sin x_{n} L^{\prime} \cosh x_{n} L^{\prime}-\cos x_{n} L^{\prime} \sinh x_{n} L^{\prime}\right)\right] .
\end{aligned}
$$

Here, $x_{n}$ is the wave number of the $n$th flexural contact resonance. The dispersion relation between $x_{n} L$ and the corresponding contact-resonance frequency $f_{n}$ is given by ${ }^{8}$

$$
\left(x_{n} L\right)^{2}=4 \pi f_{n} \frac{L^{2}}{b} \sqrt{\frac{3 \rho}{E}} .
$$

It is difficult to directly measure cantilever properties such as $E$ and $\rho$ in order to calculate $x_{n} L$ with this equation. Instead, the free-space frequency $f_{n}^{0}$ of the cantilever's $n$th flexural mode is measured. A similar relation exists between $f_{n}^{0}$ and the wave number $x_{n}^{0}$ of the $n$th flexural free-space resonance,

$$
\left(x_{n}^{0} L\right)^{2}=4 \pi f_{n}^{0} \frac{L^{2}}{b} \sqrt{\frac{3 \rho}{E}} .
$$

Moreover, for free-space vibration, $x_{n}^{0} L$ is a root of

$$
1+\cos x_{n}^{0} L \cosh x_{n}^{0} L=0 .
$$

For instance, $x_{1}^{0} L \approx 1.875$. Combining Eqs. (2) and (3),

$$
x_{n} L=x_{n}^{0} L \sqrt{\frac{f_{n}}{f_{n}^{0}}} .
$$

For the torsional resonant modes, the relation corresponding to Eq. (1) is ${ }^{10}$

$$
\frac{\kappa}{\kappa_{\text {lever }}}=-\frac{y_{n} L \cos \left(y_{n} L\right)}{\sin \left(y_{n} L_{1}\right) \cos \left(y_{n} L^{\prime}\right)} .
$$

The dispersion relation between the frequency $t_{n}$ of the $n$th torsional contact resonance and the torsional wave number $y_{n}$ is given by

$$
y_{n} L=\pi \frac{w}{b} t_{n} L \sqrt{\rho / G} .
$$

In analogy to the flexural case, we avoid direct measurements of cantilever properties such as $G$ using a relation for the free-space frequency $t_{n}^{0}$ of the $n$th torsional mode,

$$
t_{n}^{0}=\frac{(2 n-1) b}{2 L w} \sqrt{G / \rho},
$$

so that

$$
y_{n} L=\frac{(2 n-1) \pi}{2} \frac{t_{n}}{t_{n}^{0}} .
$$

These equations show how measurements of the free-space and contact-resonance frequencies can be used to determine 
the tip-sample contact stiffness. For the flexural modes, the frequency values $f_{n}^{0}$ and $f_{n}$ are used to calculate $x_{n} L$ from Eq. (4). The values of $x_{n} L$ allow Eq. (1) to be solved for $k / k_{\text {lever. }}$. Similarly, the measured torsional frequencies $t_{n}^{0}$ and $t_{n}$ yield values for $y_{n} L$ using Eq. (7), which are then used in Eq. (5) to determine $\kappa / \kappa_{\text {lever }}$. Further details of this approach, including ways to deal with the remaining variables $\left(L_{1}, h\right.$, etc.) are discussed below.

\section{B. Contact mechanics}

Given the vertical and/or the tangential contact stiffness, the elastic properties of the sample are determined by means of a model for the contact mechanics between the tip and the sample. Here, we state without proof results that are derived elsewhere. The discussion is limited to isotropic materials.

The vertical contact stiffness $k$ is determined by ${ }^{15}$

$$
k=2 a E^{*},
$$

where $a$ is the radius of contact between the tip and the sample, and $E^{*}$ is the reduced system modulus. For isotropic materials, $E^{*}$ depends on the Young's modulus $E$ and the Poisson's ratio $\nu$ of both the tip and the sample (subscript "s"),

$$
\frac{1}{E_{s}^{*}}=\frac{\left(1-\nu_{\text {tip }}^{2}\right)}{E_{\text {tip }}}+\frac{\left(1-\nu_{s}^{2}\right)}{E_{s}} .
$$

Given the definition $M=E /\left(1-\nu^{2}\right)$ for the indentation modulus $M$,

$$
\frac{1}{E_{s}^{*}}=\frac{1}{M_{\text {tip }}}+\frac{1}{M_{s}} .
$$

To determine $E^{*}$ from Eq. (8), it is necessary to know the contact radius $a$. For a flat indenter ("flat punch"), $a$ is constant. For Hertzian contact (hemispherical tip on flat sample), $a$ is given by

$$
a=\left(\frac{3 R F_{N}}{4 E^{*}}\right)^{1 / 3},
$$

where $R$ is the tip radius of curvature and $F_{N}$ is the applied static force normal to the surface. In principle, one could measure $a$ directly (for a flat punch), or determine $R$ and $F_{N}$ for a given experiment and hence determine $a$. In practice, however, accurate measurements of these properties are quite difficult. Previous work ${ }^{16,17}$ has shown how a referencing or comparison method avoids this issue. In addition, the referencing approach eliminates the need to accurately measure the cantilever spring constants $k_{\text {lever }}$ and $\kappa_{\text {lever }}$. With this approach, contact-resonance measurements are made in alternation on the unknown sample (subscript " $s$ ") and on a reference sample (subscript "ref") with known elastic properties. If the measurements are performed at the same values of the applied static force $F_{N}$, it can be shown ${ }^{16}$ that

$$
E_{s}^{*}=E_{\mathrm{ref}}^{*}\left(\frac{k_{s}}{k_{\mathrm{ref}}}\right)^{m},
$$

where $m=\frac{3}{2}$ for Hertzian contact and $m=1$ for a flat punch.
Similar equations exist for the tangential contact stiffness $\kappa,{ }^{10,18}$

$$
\kappa=8 G^{*} a,
$$

where the reduced modulus $G^{*}$ is defined as

$$
\frac{1}{G^{*}}=\frac{\left(2-\nu_{\text {tip }}\right)}{G_{\text {tip }}}+\frac{\left(2-\nu_{s}\right)}{G_{s}} .
$$

For isotropic materials, the shear modulus $G$ is defined by $G=\frac{1}{2} E /(1+\nu)$. In analogy to the normal case, we define a quantity $N=G /(2-\nu)$, so that

$$
\frac{1}{G_{s}^{*}}=\frac{1}{N_{\text {tip }}}+\frac{1}{N_{s}} .
$$

Combining Eqs. (8), (11), and (12), we obtain a relationship for the in-plane contact stiffness that corresponds to Eq. (11),

$$
G_{s}^{*}=G_{\text {ref }}^{*}\left(\frac{\kappa_{s}}{\kappa_{\text {ref }}}\right)\left(\frac{k_{s}}{k_{\text {ref }}}\right)^{m-1} .
$$

Finally, by manipulating the definitions for $M$ and $N$, it can be shown that

$$
\nu=\frac{M-4 N}{M-2 N} \text {. }
$$

The above relationships show how the individual properties $E$ and $\nu$ can be determined from contact-resonance AFM experiments. By measuring the contact-resonance frequencies of both the flexural and torsional modes under the same experimental conditions, the two contact stiffnesses $k$ and $\kappa$ can be determined. The contact stiffnesses yield values for $E^{*}$ and $G^{*}$, from which values of $M$ and $N$ for the sample are determined. Given $M$ and $N$, it is straightforward to determine $\nu, E=M\left(1-\nu^{2}\right)$, and $G=\frac{1}{2} M(1-\nu)$.

\section{EXPERIMENTAL METHODS}

\section{A. Cantilevers}

AFAM experiments are usually performed with rectangular cantilevers that are micromachined from single-crystal silicon and are relatively stiff $\left(k_{\text {lever }} \approx 50 \mathrm{~N} / \mathrm{m}\right)$. For such cantilevers, the sensitivity-the change in contact-resonance frequency with contact stiffness-is relatively large for the lowest flexural modes. ${ }^{19}$ However, the torsional contactresonance frequencies for these cantilevers are too high to measure using a typical photodiode detector with a rolloff of 2-3 MHz. Previous contact-resonance torsional experiments were performed with more compliant cantilevers $\left(k_{\text {lever }} \approx 0.1\right.$ to $\left.1 \mathrm{~N} / \mathrm{m}\right) .{ }^{10}$ Although for these cantilevers the torsional contact-resonance frequencies are low enough to be detected, the sensitivity of their flexural modes is poor. The geometry of the particular cantilever that we used was chosen to maximize the response of both the flexural and torsional modes as much as possible.

The graphs in Fig. 2 can be used to understand this issue better. Figure 2(a) shows the change in contact-resonance frequency $f_{n}$ as a function of vertical contact stiffness $k$ for the lowest two flexural modes. The frequency is normalized to the frequency of the first free-space mode $f_{1}^{0}$, and the 

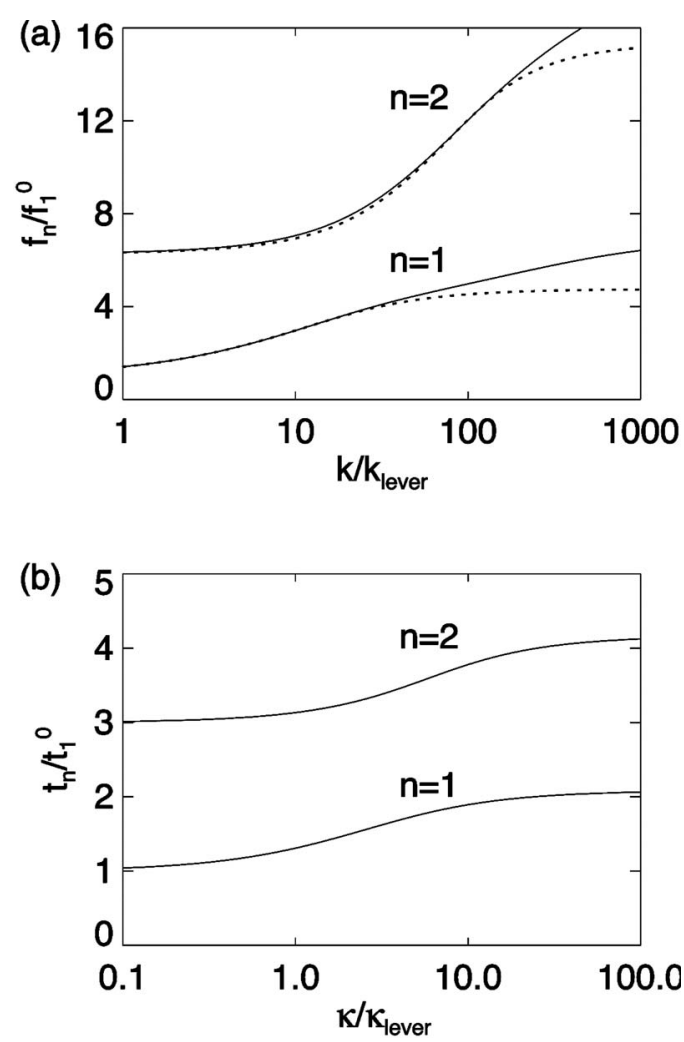

FIG. 2. Frequency as a function of contact stiffness for (a) flexural and (b) torsional cantilever vibrations. The frequencies are normalized to the corresponding fundamental free-space frequency, i.e., $f_{1}^{0}$ for the flexural modes and $t_{1}^{0}$ for torsional modes. The vertical contact stiffness $k$ is normalized to the cantilever flexural spring constant $k_{\text {lever }}$, while the tangential contact stiffness $\kappa$ is normalized to the constant $\kappa_{\text {lever }}$ defined in the text. The curves in (a) were calculated by assuming $L_{1} / L=0.96, h / L_{1}=0.1, \alpha=11^{\circ}$, and $\kappa / k=0.9$. The dotted lines were calculated by assuming $\kappa=\alpha=h=0$ and $L_{1} / L=0.96$. The curves in (b) for the torsional modes were calculated by assuming $L_{1} / L=0.96$. Only the first two modes of each type of resonance $(n=1,2)$ are shown.

contact stiffness is normalized to the cantilever stiffness $k_{\text {lever}}$. The curves were generated from Eq. (1) assuming $L_{1} / L=0.96, h / L_{1}=0.1, \alpha=11^{\circ}$, and $\kappa / k=0.9$. To show the contribution of the lateral spring, the dotted lines were calculated assuming $\kappa=\alpha=h=0$ (i.e., no lateral spring) and $L_{1} / L=0.96$. Figure 2(b) shows the change in the torsional contact-resonance frequency $t_{n}$ as a function of tangential contact stiffness $\kappa$ for the lowest two torsional modes. The frequency is normalized to the frequency of the first freespace mode $t_{1}^{0}$, and the contact stiffness is normalized to the cantilever stiffness $\kappa_{\text {lever }}$. These curves were generated from Eq. (5) with $L_{1} / L=0.96$.

Figure 2 shows that the dependence of the flexural and torsional modes on the corresponding contact stiffness is qualitatively similar. When the contact stiffness is low, the contact-resonance frequency remains close to its free-space value. As the contact stiffness increases, the slope of the curve gradually increases, and thus the sensitivity increases. For larger values of the contact stiffness, the tip-sample coupling approaches a "pinned" condition, and the frequency changes very little with contact stiffness. At this point, the contact-resonance frequency of the $n$th mode approaches the free-space frequency of the $(n+1)$ th mode.
To achieve accurate measurements of elastic properties, we wish to operate in a region of high sensitivity. In such regions, small changes in contact stiffness produce measurable changes in the contact-resonance frequency. We want to maximize the sensitivity of both the flexural and torsional modes in order to accurately measure $E$ and $\nu$ individually. The sensitivity of a given cantilever can be predicted by estimating the expected experimental values of the normalized contact stiffnesses $k / k_{\text {lever }}$ and $\kappa / \kappa_{\text {lever. }}$. This is achieved by first calculating the contact radius $a$ using Eq. (10) with typical values of $R(\sim 10-50 \mathrm{~nm})$ and $F_{N}(\sim 0.1-1 \mu \mathrm{N})$. Values for $k$ and $\kappa$ are then calculated from $a$ with Eqs. (8) and (12), respectively, for the material of interest. The cantilever flexural and torsional spring constants $k_{\text {lever }}$ and $\kappa_{\text {lever }}$ are estimated from nominal values of the cantilever dimensions and material properties. We performed such calculations for several cantilever geometries, and chose the one for which both $k / k_{\text {lever }}$ and $\kappa / \kappa_{\text {lever }}$ fell closest to the regions of high sensitivity.

Based on these considerations, the cantilever used for these experiments had nominal dimensions of length $L$ $=230 \pm 5 \mu \mathrm{m}$, width $w=40 \pm 3 \mu \mathrm{m}$, and thickness $b$ $=3.0 \pm 0.5 \mu \mathrm{m}$. The nominal tip height $h$ was $20-25 \mu \mathrm{m}$. The flexural spring constant had a nominal value $k_{\text {lever }}$ $=3.5 \pm 2 \mathrm{~N} / \mathrm{m}$. The free-space frequencies of the cantilever were measured to be $t_{1}^{0}=797.18 \pm 0.02 \mathrm{kHz}$ and $t_{2}^{0}$ $=2649.7 \pm 0.1 \mathrm{kHz}$ for the lowest two torsional modes, and $f_{1}^{0}=72.91 \pm 0.02 \mathrm{kHz}$ and $f_{2}^{0}=495.94 \pm 0.02 \mathrm{kHz}$ for the lowest two flexural modes. Note that the measured values of the cantilever's free resonances differ from those predicted by the idealized beam model. For torsional modes, the theoretical ratio of the second to first free frequencies is $t_{2}^{0} / t_{1}^{0}=3$ [see Eq. (6)]. For flexural modes, the corresponding ratio is $f_{2}^{0} / f_{1}^{0}=(4.6941 / 1.8751)^{2}=6.27$ (see, for instance, Ref. 14). The actual ratios measured for this cantilever were $t_{2}^{0} / t_{1}^{0}$ $=3.3$ and $f_{2}^{0} / f_{1}^{0}=6.8$. This deviation is larger than usually observed for AFAM cantilevers. However, this was the only cantilever we could identify with sufficient sensitivity in both the flexural and torsional modes within the detection bandwidth of our AFM instrument. As discussed below, the effects of this discrepancy can be seen in more than one aspect of the data analysis.

It is possible to adjust the experimental conditions somewhat, particularly $F_{N}$, in order to enhance the response of a given cantilever for a given material. However, the choice of operating conditions is severely limited by the range of commercially available cantilevers. The accuracy of this method might be improved by use of custom-fabricated cantilevers with a geometry intentionally designed for both flexural and torsional sensitivity.

\section{B. Specimens}

Because quantitative measurements with torsional modes have not been demonstrated previously, we used materials whose properties were already known. In this way, the validity of our approach could be checked. The two specimens were a fused silica $\left(\mathrm{SiO}_{2}\right)$ plate with dimensions 14.6 $\times 14.6 \times 0.8 \mathrm{~mm}^{3}$, and a disk of borosilicate crown glass 
TABLE I. Properties of materials used in the experiments. All quantities except Poisson's ratio $\nu$ are given in GPa. The first two rows contain the values assumed for the two materials based on pulse-echo ultrasonic experiments and literature surveys. The last two rows show the experimental contact-resonance AFM results for the glass specimen with the fused silica $\left(\mathrm{SiO}_{2}\right)$ specimen used as the reference material. Values are given assuming Hertzian $(m=3 / 2)$ and flat-punch $(m=1)$ contact mechanics.

\begin{tabular}{ccccccc}
\hline \hline Material & Source & $M$ & $N$ & $\nu=\frac{M-4 N}{M-2 N}$ & $G=N(2-\nu)$ & $E=M\left(1-\nu^{2}\right)$ \\
\hline \multirow{2}{*}{$\mathrm{SiO}_{2}$} & Literature & 74.9 & 17.0 & 0.171 & 31.1 & 72.7 \\
Glass & Literature & 84.7 & 18.7 & 0.206 & 33.6 & 81.1 \\
& Expt. $m=1$ & $81 \pm 5$ & $18 \pm 2$ & $0.21 \pm 0.11$ & $32 \pm 5$ & $76 \pm 6$ \\
& Expt. $m=3 / 2$ & $85 \pm 8$ & $19 \pm 3$ & $0.17 \pm 0.16$ & $35 \pm 8$ & $79 \pm 10$ \\
\hline \hline
\end{tabular}

with diameter $12.5 \mathrm{~mm}$ and thickness $1 \mathrm{~mm}$. The fused silica sample was taken as the reference material, and the glass was considered to be the "unknown" material. Based on pulseecho ultrasonic measurements, ${ }^{20}$ we used the values $E_{\mathrm{SiO}_{2}}$ $=72.7 \mathrm{GPa}$ and $\nu=0.171$ for fused silica, and $E_{\text {glass }}$ $=81.1 \mathrm{GPa}$ and $\nu_{\text {glass }}=0.206$ for the glass sample. These values are nearly identical to nominal values quoted by vendors. The properties of the two specimens are summarized in the first two rows of Table I. For each material, the table contains Young's modulus $E$ and Poisson's ratio $\nu$. Also shown are the values of the indentation modulus $M$, the shear modulus $G$, and the quantity $N$ calculated from these values of $E$ and $\nu$.

One reason that these specimens were chosen is their isotropic, homogeneous nature. Another reason derives from the results of AFAM flexural-mode experiments. It has been shown that the best results are obtained when the values of $M_{\text {ref }}$ and $M_{s}$ are not dramatically different. ${ }^{17,18,21}$ Presumably, a similar principle applies for torsional measurements and the relative values of $N_{\text {ref }}$ and $N_{s}$. For our specimens, $M_{\mathrm{SiO}_{2}}$ $=74.9 \mathrm{GPa}$ and $M_{\text {glass }}=84.7$, while $N_{\mathrm{SiO}_{2}}=17.0 \mathrm{GPa}$ and $N_{\text {glass }}=18.7 \mathrm{GPa}$. These values are sufficiently alike that we expect accurate measurement results.

\section{Measurement procedure}

The equipment and experimental procedure are similar to those described in detail elsewhere. ${ }^{18}$ The description here relates mainly to the detection of torsional modes. Figure 3 shows a schematic of the experimental components. To ex-

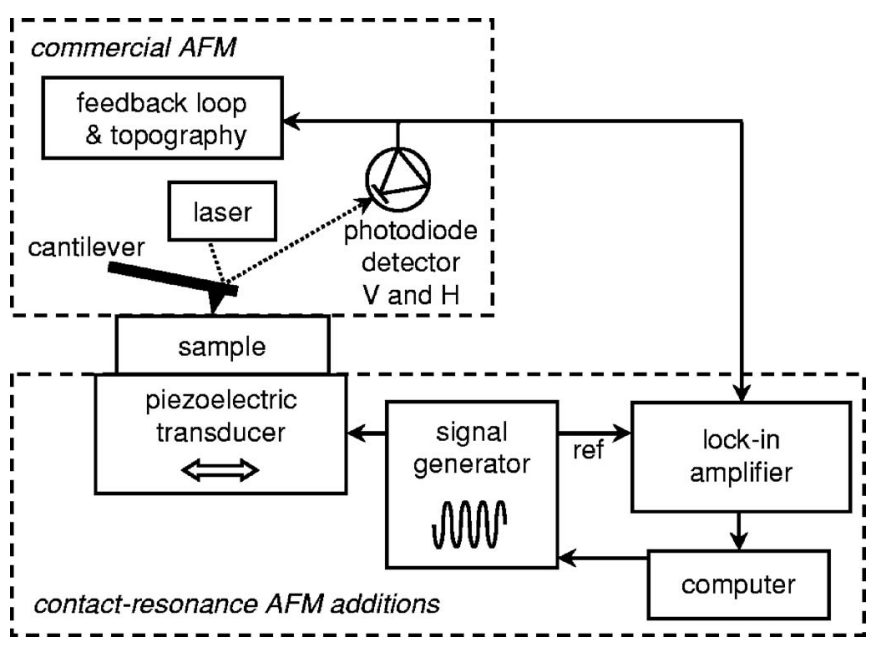

FIG. 3. Block diagram of experimental AFAM apparatus. cite the resonant cantilever vibrations, commercial ultrasonic transducers were used. The transducers contained piezoelectric elements designed to generate shear (transverse) waves, and therefore produced in-plane displacements. Each specimen was bonded to a separate transducer with honey. Honey was used as an acoustic couplant because it is a highly viscous liquid that supports transverse waves, but is easy to remove with water when the sample is unmounted. ${ }^{22} \mathrm{~A}$ drop of honey was placed on the transducer, and the sample placed on top. Slight pressure was applied by hand to evenly distribute the bonding layer. The mounted samples were left overnight before the measurements were made to account for any slow flow of the bond. The transducers were mounted on the AFM stage so that the displacement was perpendicular to the long axis of the cantilever. The transducer was excited with a continuous sine wave voltage by a signal generator (frequency $\sim 0.05-3.0 \mathrm{MHz}$, amplitude $\sim 25-100 \mathrm{mV}$ ). The amplitude of the cantilever vibration as a function of excitation frequency was determined with a lock-in amplifier. It is important to note that these measurements require access to the unfiltered, high-frequency signal from the AFM photodiode detector for both the vertical and horizontal channels. To detect the flexural vibrations, we used the vertical photodiode signal for the lock-in input. The horizontal signal was used to detect the torsional modes.

To measure the contact-resonance frequencies, the tip was brought into contact with the sample. For each sample position, measurements were made at four different values of the cantilever deflection $\delta$. Using $k_{\text {lever }} \approx 4 \mathrm{~N} / \mathrm{m}$, the values $\delta=60,90,120$, and $150 \mathrm{~nm}$ correspond to static forces $F_{N}$ $=k_{\text {lever }} \delta \approx 250-600 \mathrm{nN}$. At each applied force, contactresonance spectra were acquired for the two lowest flexural modes and for the two lowest torsional modes. This process was repeated several times in alternation on the unknown (glass) and reference $\left(\mathrm{SiO}_{2}\right)$ specimens. Note that, in principle, excitation of the flexural resonances requires an outof-plane displacement by the transducer. However, we discovered that with our experimental configuration, in-plane displacements excited small, but detectable, flexural resonance signals.

\section{DATA ANALYSIS}

The measurement procedure described above yields values for the frequencies of the two lowest flexural and two lowest torsional resonant modes for each specimen. The basic concepts by which these frequencies are used to deter- 
mine the elastic properties of the unknown specimen are outlined in Sec. II. However, there are several different ways that the models in Sec. II can be implemented in practice. One reason for this is the relatively large number of parameters involved $\left(k / k_{\text {lever }}, k / k_{\text {lever }}, h / L_{1}, L_{1} / L\right.$, etc.). Ideally, analysis would consist of a multiparameter optimization that simultaneously determines the best-fit values of all parameters. However, for this proof-of-principle demonstration, we chose a more straightforward approach that is simpler to implement. This method consists of the following steps.

(1) Flexural-mode analysis to determine $k / k_{\text {lever }}$ and $L_{1} / L$. First, we analyzed the frequency data for the flexural modes using Eqs. (1) and (4). Equation (4) was used to determine $x_{n} L$ from the free-space and contactresonance frequencies. There are five other variables in Eq. (1):

(a) the cantilever tilt angle $\alpha$;

(b) the ratio of the tangential to vertical contact stiffness $\kappa / k$;

(c) the ratio of the tip height to tip position $h / L_{1}$;

(d) the relative tip position $L_{1} / L$; and

(e) the normalized vertical contact stiffness $k / k_{\text {lever }}$.

To reduce the number of variables, we used fixed values for parameters (a), (b), and (c). The cantilever tilt $\alpha$ is constant for a given atomic force microscope; we used $\alpha=11^{\circ}$. For the tip height, we used $h / L_{1}=0.1$, based on the nominal cantilever dimensions. For the tangential-tovertical ratio $\kappa / k$, we used the value calculated for the reference material using $\kappa / k=4 G^{*} / E^{*}$ [see Eqs. (8) and (12)]. With $M_{\text {tip }}=165.1 \mathrm{GPa}$ and $N_{\text {tip }}=37.5 \mathrm{GPa}$ for the silicon tip (see below), $\kappa / k=0.91$ for $\mathrm{SiO}_{2}$. Using these values as fixed input parameters, we solved Eq. (1) for the normalized vertical contact stiffness $k / k_{\text {lever }}$ as a function of relative tip position $L_{1} / L$. As was found in previous work, ${ }^{8,16,17}$ the two modes have the same value of $k / k_{\text {lever }}$ for only one physically realistic value of $L_{1} / L$. These values of $k / k_{\text {lever }}$ and $L_{1} / L$ were taken as the solution.

(2) Torsional-mode analysis to determine $\kappa / \kappa_{\mathrm{lever}}$. Next, we analyzed the frequency data for the torsional modes with Eqs. (5) and (7). We used Eq. (7) to determine $y_{n} L$ from the free-space and contact-resonance frequencies. Equation (5) has two other variables: the normalized tangential contact stiffness $\kappa / \kappa_{\text {lever }}$ and the relative tip position $L_{1} / L$. For the tip position $L_{1} / L$, we used the average value determined from the flexural analysis in step 1 for all of the data. Torsional-mode analysis then consists of solving Eq. (5) for $k / k_{\text {lever }}$ for this value of $L_{1} / L$ for each torsional mode $(n=1,2)$. For these data, it was found that the two modes do not always share a common value of $\kappa / \kappa_{\text {lever }}$ for physically realistic values of $L_{1} / L$. The reason for this behavior is not clear, but is likely due to differences between the actual cantilever and the behavior predicted by the idealized model. One justification for this explanation is the discrepancy noted above between the measured and predicted spacing of the free torsional modes. Therefore, for the value of $\kappa / \kappa_{\text {lever }}$ we used the average of the values for the two individual modes.

(3) Contact-stiffness analysis to determine $E^{*}$ and $G^{*}$. Steps 1 and 2 yield values of the normalized contact stiffnesses $k / k_{\text {lever }}$ and $\kappa / \kappa_{\text {lever }}$ for both the unknown and reference specimens. From the contact-stiffness values, $E_{s}^{*}$ and $G_{s}^{*}$ were calculated using Eqs. (11) and (14). Each set of measurements on the unknown material was compared to two sets of reference measurements: those made directly before, and those made directly afterwards. Thus, each measurement of four contactresonance frequencies on the test material yielded two values of $E_{s}^{*}$ and $G_{s}^{*}$.

If desired, a revised value of $\kappa / k$ can be calculated from the values of $E_{s}^{*}$ and $G_{s}^{*}$ for the unknown material, and compared to the assumed value. The revised value of $\kappa / k$ can be used to repeat the analysis of $k / k_{\text {lever }}$ for the unknown specimen. This iteration can be repeated until $\kappa / k$ converges, and final values for $E_{s}^{*}$ and $G_{s}^{*}$ are calculated. We found that for these data, only two or three iterations were needed for $\kappa / k$ to converge.

(4) Calculation of elastic properties. From the values of $E_{s}^{*}$ and $G_{s}^{*}$, values for $M_{s}$ and $N_{s}$ were calculated using Eqs. (9) and (13), respectively. Once the experimental values for $M_{s}$ and $N_{s}$ were obtained, Poisson's ratio $\nu_{s}$ for the unknown material was determined using Eq. (15). Finally, values for the shear modulus $G$ and Young's modulus $E$ were determined using the relations between $E, \nu, M$, and $N$ given above.

In these calculations, values for the properties $M_{\text {tip }}$ and $N_{\text {tip }}$ of the $\langle 100\rangle$ silicon tip are needed. The theory to determine the indentation modulus $M$ for an anisotropic material has been published. ${ }^{23}$ Based on that work, we used $M_{\text {tip }}$ $=165.1 \mathrm{GPa}$. However, the corresponding theoretical framework for the quantity $N_{\text {tip }}$ in anisotropic materials has not been developed. We used the value $N_{\text {tip }}=37.5 \mathrm{GPa}$ obtained from $N=G /(2-\nu)$ with $G_{\mathrm{Si}}=66.6 \mathrm{GPa}$ and $\nu_{\mathrm{Si}}=0.223$. These values of $G$ and $\nu$ were obtained from the Voigt-Reuss averages for randomly oriented (isotropic), polycrystalline silicon from the elastic constants of single-crystal silicon. ${ }^{24}$ The difference between the assumed and actual values of $N_{\text {tip }}$ (and $M_{\text {tip }}$ ) will affect our final results. However, changing $N_{\text {tip }}$ by as much as $20 \%$ changed the results for $M_{s}$ and $N_{s}$ by only $1 \%$ or $2 \%$. Improved values of $N_{\text {tip }}\left(\right.$ and/or $\left.M_{\text {tip }}\right)$ might be obtained using an approach that utilizes two reference materials. $^{21}$

\section{RESULTS AND DISCUSSION}

The experimental procedure described above was performed on the two specimens, resulting in a total of 20 ratios between the unknown and reference materials. Table II contains representative data to illustrate the experimental and analysis procedure. Given in the table are the measured contact-resonance frequencies $f_{1}, f_{2}, t_{1}$, and $t_{2}$. Also shown are the vertical stiffness $k / k_{\text {lever }}$ and tip position $L_{1} / L$ determined from analysis of the flexural modes, and the tangential 
TABLE II. Examples of measured contact-resonance frequencies for a cantilever with free-space frequencies $f_{1}^{0}=72.91 \pm 0.02 \mathrm{kHz}, f_{2}^{0}=495.94 \pm 0.02 \mathrm{kHz}, t_{1}^{0}=797.18 \pm 0.02 \mathrm{kHz}$, and $t_{2}^{0}=2649.7 \pm 0.1 \mathrm{kHz}$. The frequency measurements were made with a resolution of $0.1 \mathrm{kHz}$. Included are the values obtained for the normalized contact stiffnesses $k / k_{\text {lever }}$ and $\kappa / \kappa_{\text {lever }}$ and the cantilever parameters $L_{1} / L$ by use of the analysis method described in the text.

\begin{tabular}{ccccccccc}
\hline \hline Material & $\delta(\mathrm{nm})$ & $f_{1}(\mathrm{kHz})$ & $f_{2}(\mathrm{kHz})$ & $\kappa / \kappa_{\text {lever }}$ & $L_{1} / L$ & $t_{1}(\mathrm{kHz})$ & $t_{2}(\mathrm{kHz})$ & $\kappa / \kappa_{\text {lever }}$ \\
\hline $\mathrm{SiO}_{2}$ & 60 & 428.8 & 1191.2 & 228.87 & 0.9340 & 1409.8 & 2774.8 & 2.93 \\
& 90 & 430.3 & 1199.7 & 238.97 & 0.9349 & 1430.2 & 2780.1 & 3.18 \\
& 120 & 432.4 & 1204.4 & 238.93 & 0.9326 & 1450.5 & 2788.4 & 3.49 \\
& 150 & 433.1 & 1208.0 & 243.26 & 0.9329 & 1454.6 & 2791.2 & 3.56 \\
Glass & 60 & 429.6 & 1203.8 & 251.42 & 0.9386 & 1418.3 & 2777.4 & 3.03 \\
& 90 & 433.2 & 1212.4 & 252.33 & 0.9349 & 1446.6 & 2784.8 & 3.42 \\
& 120 & 435.2 & 1220.8 & 261.55 & 0.9348 & 1469.2 & 2796.3 & 3.81 \\
$\mathrm{SiO}_{2}$ & 150 & 437.5 & 1230.7 & 273.28 & 0.9348 & 1488.4 & 2800.6 & 4.17 \\
& 60 & 429.1 & 1197.4 & 239.43 & 0.9363 & 1428.8 & 2779.2 & 3.16 \\
& 90 & 432.2 & 1210.4 & 252.89 & 0.9361 & 1435.8 & 2784.6 & 3.27 \\
& 120 & 434.1 & 1214.4 & 252.21 & 0.9339 & 1467.6 & 2792.6 & 3.77 \\
& 150 & 435.2 & 1218.8 & 256.58 & 0.9337 & 1477.0 & 2799.2 & 3.96 \\
\hline \hline
\end{tabular}

stiffness $\kappa / \kappa_{\text {lever }}$ determined from the torsional-mode analysis. These values were obtained with the procedure described in Sec. IV.

Values for the tip position parameter $L_{1} / L$ calculated in the data analysis ranged from approximately 0.93 to 0.95 . The average value of all of the data was $L_{1} / L$ $=0.939 \pm 0.005$. For comparison, $L_{1} / L$ was measured directly by examining the cantilever in a calibrated optical microscope. Measurements of the cantilever dimensions in plan view yielded $L_{1} / L=0.977 \pm 0.003$. In other AFAM experiments, similar discrepancies between the calculated and measured values of $L_{1} / L$ have been observed. ${ }^{18}$ The most likely explanation for the discrepancy is the deviation of the cantilever's actual behavior from that predicted by the idealized beam model. $^{14}$

Table II also shows that both the vertical and tangential contact stiffnesses $k$ and $\kappa$ depend on the applied force $F_{N}$. In previous work that examined the force dependence of $k$ in AFAM experiments, ${ }^{25}$ it was found that the actual behavior corresponded to a tip shape intermediate between a hemisphere and a flat punch. The data were fit to $k \propto F_{N}^{\beta}$, where $\beta$ varied between 0 (flat punch) and $1 / 3$ (hemisphere). Similar analysis of the current data yields $\beta \approx 0.05-0.15$ for $k$, consistent with the previous results. Simple contact-mechanics models predict that the force dependence of $\kappa$ should be the same as that of $k$, because both depend in the same way on the contact radius $a$ [see Eqs. (8), (10), and (12)]. In several of the data sets, $\beta \approx 0.10-0.16$ for $\kappa$, similar to the corresponding values for $k$. However, for the data in Table II, $\beta$ $\approx 0.22-0.35$ for $\kappa$. The reason for the variation in $\beta$ between data sets is not clear. Further experiments are necessary to study the force dependence of $\kappa$ in detail.

From data such as those in Table II, experimental values for the properties of the glass specimen were calculated. The results are summarized in the last two rows of Table I, which show the values obtained assuming Hertzian $\left(m=\frac{3}{2}\right)$ and flatpunch $(m=1)$ contact mechanics. The values obtained with these two models represent the upper and lower limits of the measured properties. Shown are the average values of the indentation modulus $M$ and the quantity $N$ obtained from the contact-resonance experiments. Also included are the calculated values of Young's modulus $E$, Poisson's ratio $\nu$, and the shear modulus $G$. These values are averages of 20 individual values calculated from $M$ and $N$ for each measurement. (Slightly different results are obtained if one calculates a single value of $E, \nu$, and $G$ using the average values of $M$ and $N$.) The measurement uncertainties represent 1 standard deviation due to scatter in the 20 individual measurements.

A comparison of the values in Table I reveals that our experimental results are in good agreement with the assumed values. All of the values agree within the measurement uncertainty. All of the glass sample's properties except $E$ are bracketed by the measured values calculated for $m=1$ and $m=\frac{3}{2}$. The uncertainties in the quantities $M$ and $N$, which are directly determined from the measurements, are approximately $5 \%$ to $15 \%$. In comparison, the typical uncertainty for contact-resonance measurements of $M$ using stiffer cantilevers is approximately $5 \%$ to $10 \%$. The uncertainties for the other properties $(E, \nu$, and $G)$ are larger, because they are calculated from combinations of $M$ and $N$. For instance, as seen in Eq. (15), $\nu$ is calculated from the ratio of two differences in $M$ and $N$. This uncertainty is reflected in the uncertainties in $E$ and $G$, which are calculated from $\nu$. As mentioned above, a more sophisticated analysis procedure could be developed. An analysis approach that optimizes the system parameters for each measurement is likely to reduce the scatter in the individual measurements. Refinements to the experimental approach might also reduce the measurement uncertainty.

\section{SUMMARY AND CONCLUSION}

We have described methods for the quantitative determination of elastic properties with contact-resonance AFM. Measurements of both the flexural and torsional contactresonance frequencies make it possible to determine separate values for Young's modulus $E$ and Poisson's ratio $\nu$ at the same time with one technique. Contact-resonance AFM methods are relatively straightforward to implement with a 
commercial atomic force microscope. Moreover, the referencing approach means that tedious, specialized measurements of cantilever properties such as $k_{\text {lever }}$ or $R$ are not necessary. Refinements to the method described here could improve measurement sensitivity and precision. Further work is also necessary to investigate the achievable limits of spatial resolution. Nonetheless, these initial results demonstrate the potential of contact-resonance AFM methods for accurate nanomechanical measurements.

\section{ACKNOWLEDGMENTS}

J.A.T. gratefully acknowledges the National Science Foundation for partial support of this work. We are grateful to L. Yang (University of Nebraska-Lincoln) and $\mathrm{M}$. Kopycinska-Müller (NIST) for valuable discussions. We thank C. Su (Veeco) for advice in making the necessary modifications to access the horizontal photodiode signal.

\footnotetext{
${ }^{1}$ Instrumentation and Metrology for Nanotechnology, Report of the National Nanotechnology Initiative Workshop, 27-29 January 2004, Gaithersburg, MD; http://www.nano.gov/NNI_Instrumentation_Metrology_rpt. pdf

${ }^{2}$ International Technology Roadmap for Semiconductors, http:// public.itrs.net (2005).

${ }^{3}$ W. C. Oliver and G. M. Pharr, J. Mater. Res. 7, 1564 (1992).

${ }^{4}$ W. D. Nix, Metall. Trans. A 20A, 2217 (1989).

${ }^{5}$ B. N. Lucas, J. C. Hay, and W. C. Oliver, J. Mater. Res. 19, 58 (2004).

${ }^{6}$ T. Drobek, R. W. Stark, and W. M. Heckl, Phys. Rev. B 64, 04540
}

(2001).

7O. Piétrement and M. Troyon, Surf. Interface Anal. 31, 1060 (2001).

${ }^{8}$ U. Rabe, S. Amelio, E. Kester, V. Scherer, S. Hirsekorn, and W. Arnold, Ultrasonics 38, 430 (2000).

${ }^{9}$ K. Yamanaka and S. Nakano, Appl. Phys. A: Mater. Sci. Process. 66, S313 (1998).

${ }^{10}$ M. Reinstädtler, T. Kasai, U. Rabe, B. Bhushan, and W. Arnold, J. Phys. D 38, R269 (2005).

${ }^{11}$ R. W. Carpick and M. Salmeron, Chem. Rev. 97, 1163 (1997).

${ }^{12}$ Y. Song and B. Bhushan, J. Appl. Phys. 97, 083533 (2005).

${ }^{13}$ U. Rabe, J. Turner, and W. Arnold, Appl. Phys. A: Mater. Sci. Process. 66, S277 (1998)

${ }^{14} \mathrm{U}$. Rabe, in Applied Scanning Probe Methods II, edited by B. Bhushan and H. Fuchs (Springer, New York, 2006), pp. 39-70.

${ }^{15}$ K. L. Johnson, Contact Mechanics (Cambridge University Press, Cambridge, 1985), pp. 91-95.

${ }^{16}$ U. Rabe, S. Amelio, M. Kopycinska, S. Hirsekorn, M. Kempf, M. Göken, and W. Arnold, Surf. Interface Anal. 33, 65 (2002).

${ }^{17}$ D. C. Hurley, K. Shen, N. M. Jennett, and J. A. Turner, J. Appl. Phys. 94, 2347 (2003).

${ }^{18}$ R. W. Carpick, D. F. Ogletree, and M. Salmeron, Appl. Phys. Lett. 70, 1548 (1997)

${ }^{19}$ J. A. Turner and J. S. Wiehn, Nanotechnology 12, 322 (2001).

${ }^{20}$ E. P. Papadakis, in Physical Acoustics, edited by W. P. Mason and R. N. Thurston (Academic, San Diego, 1976), Vol. 12, p. 277.

${ }^{21}$ G. Stan and W. Price, Rev. Sci. Instrum. 77, 103707 (2006).

${ }^{22}$ J. Krautkrämer and H. Krautkrämer, Ultrasonic Testing of Materials (Springer, Berlin, 1990), p. 271.

${ }^{23}$ J. J. Vlassak and W. D. Nix, Philos. Mag. A 67, 1045 (1993).

${ }^{24}$ H. J. McSkimin and P. A. Andreatch, J. Appl. Phys. 35, 2161 (1964).

${ }^{25}$ M. Kopycinska-Müller, R. H. Geiss, and D. C. Hurley, Ultramicroscopy 106, 466 (2006) 